\title{
High Autophagy in the Naked Mole Rat may Play a Significant Role in Maintaining Good Health
}

\author{
Shanmin Zhao ${ }^{a, d}$ Lifang Lin ${ }^{a, d}$ Guanghan Kan ${ }^{b, d}$ Chang Xuc,d Qiu Tang ${ }^{c}$ \\ Chenlin Yu ${ }^{a}$ Wei Sun ${ }^{a}$ Liping Cai ${ }^{a}$ Chen Xu Shufang Cui ${ }^{a}$ \\ aLaboratory Animal Centre of the Second Military Medical University, Shanghai, bChina Astronaut \\ Research and Training Center, Beijing, 'School of kinesology, Shanghai University of Sport, Shanghai, \\ China; ${ }^{\mathrm{d} T h e s e}$ authors contributed equally to this work
}

\section{Key Words}

Autophagy • Apoptosis • Macroautophagy • Hepatic stellate cell

\begin{abstract}
Background/Aims: The maximum lifespan of the naked mole rat is over 28.3 years, which exceeds that of any other rodent species, suggesting that age-related changes in its body composition and functionality are either attenuated or delayed in this extraordinarily longlived species. However, the mechanisms underlying the aging process in this species are poorly understood. In this study, we investigated whether long-lived naked mole rats display more autophagic activity than short-lived mice. Methods: Hepatic stellate cells isolated from naked mole rats were treated with $50 \mathrm{nM}$ rapamycin or $20 \mathrm{mM}$ 3-methyladenine (3-MA) for 12 or $24 \mathrm{~h}$. Expression of the autophagy marker proteins LC3-II and beclin 1 was measured with western blotting and immunohistochemistry. The induction of apoptosis was analyzed by flow cytometry. Results: Our results demonstrate that one-day-old naked mole rats have higher levels of autophagy than one-day-old short-lived C57BL/6 mice, and that both adult naked mole rats (eight months old) and adult C57BL/6 mice (eight weeks old) have high basal levels of autophagy, which may be an important mechanism inhibiting aging and reducing the risk of age-related diseases. Conclusion: Here, we report that autophagy facilitated the survival of hepatic stellate cells from the naked mole rat, and that treatment with 3-MA or rapamycin increased the ratio of apoptotic cells to normal hepatic stellate cells.
\end{abstract}




\section{Introduction}

Naked mole rats, Heterocephalus glaber (Family: Bathyergidae), are small burrowing rodents native to northeast Africa. They can live for over 30 years in captivity, remaining in good health and cancer-free well into their third decade of life. The air in naked mole rat burrows is usually low in $\mathrm{O}_{2}$ (approximately 8\%) and high in $\mathrm{CO}_{2}$ (approximately 10\%) because of the poor gas exchange through soil and because several naked mole rats share this limited air supply. Therefore, these animals live in a chronically low-oxygen environment, but are remarkably resistant to hypoxia [1]. Autophagy is a catabolic mechanism by which cells recycle amino acids and other intracellular nutrients to obtain energy. This process is evolutionarily conserved in the eukaryotes, ranging from yeast to mammals [2]. The activation of macroautophagy is a common feature among all long-lived mutant Caenorhabditis elegans [3-5]. The naked mole rat is known to have the longest lifespan among rodents, with a maximum lifespan exceeding 28.3 years [6]. Studies of senescent avian and mammalian fibroblasts have suggested that cells obtained from long-lived birds maintain higher levels of autophagosomal and lysosomal enzymes, which are downregulated in older mammalian cell lines. We hypothesized that increased autophagy may protect the naked mole rat from several forms of stress.

The formation of autophagosomes requires a process termed "light chain 3 (LC3) modification". LC3, a pacemaker in the autophagic cascade, is currently the most reliable cellular marker of autophagy activation [7-9]. During this process, LC3-II is formed by the lipidation of LC3-I and becomes incorporated into the autophagosomal membrane, and this conversion from LC3-I to LC3-II is used as an index of autophagy [10]. Beclin 1, another pacemaker, is involved in the formation of preautophagosomal structures.

Hepatic stellate cells comprise approximately one-third of the nonparenchymal cell population and $\sim 15 \%$ of the total number of resident cells in the normal liver. This cell type is a remarkably versatile mesenchymal cell that is vital to hepatocellular function and the liver's response to injury [11]. Currently, the most commonly used inducer of autophagy is rapamycin, an allosteric inhibitor of target of rapamycin C1 (TORC1). 3-Methyladenine (3-MA) can effectively block an early stage of autophagy by inhibiting class III phosphatidylinositide 3-kinases and also inhibits class I phosphatidylinositide 3-kinases [12]. In this study, we compared the expression of the autophagy-related proteins, LC3 and beclin 1, in naked mole rats and C57BL/6 mice to determine whether long-lived naked mole rats display more autophagic activity than short-lived mice. We then evaluated the autophagy rate and the survival of naked mole rat hepatic stellate cells treated with 3-MA or rapamycin.

\section{Materials and Methods}

\section{Ethics statement}

Five one-day-old C57BL/6 mice and five adult C57BL/6 mice (eight weeks old) were purchased from SLAC Laboratory Animal Co., Ltd. (Shanghai, China). Naked mole rats were purchased from the Department of Zoology at the University of Cape Town and maintained at the Laboratory Animal Center of the Second Military Medical University. The handling of all animals and all study procedures complied with the current Chinese regulation GB14925-2001: Laboratory animal requirements of environment and housing facilities (Chinese version). The complete protocol was reviewed and approved by the Institutional Animal Care and Use Committee of the Second Military Medical University. All surgery was performed under sodium pentobarbital anesthesia, and all efforts were made to minimize the suffering of the animals.

\section{Extraction of total protein from tissues}

One-day-old naked mole rats, adult naked mole rats (eight months old), one-day-old C57BL/6 mice, and adult C57BL/6 mice (eight weeks old) were anesthetized with isoflurane, killed by cardiac exsanguination, and their liver, brain, and kidney tissues were immediately excised. The tissues were weighed and the wet 
tissue weights were recorded. The tissue samples were cut into small pieces with a scalpel, homogenized on ice with a homogenizer, and subjected to total protein extraction using Tissue Protein Extraction Reagent (Wuhan Boster Biotech, Wuhan, China). A wet transfer method was used to transfer the proteins electrophoretically from the native gel to nitrocellulose membranes, which were then probed with antibeclin 1 antibody (1:1000; Abcam, Cambridge, UK) or anti-LC3 antibody (1:1000; Cell Signaling Technology, Shanghai, China). The blots were stripped and reprobed with anti- $\beta$-actin antibody (1:2000; Wuhan Boster Biotech, Wuhan, China) to confirm equal protein loading. The bands were visualized with the Kodak Gel Logic 4000 R Imaging System (Carestream, USA).

\section{Transmission electron microscopic examination}

One-day-old naked mole rats and one-day-old C57BL/6 mice were deeply anesthetizedand transcardially perfused with $0.1 \mathrm{M}$ phosphate-buffered saline (PBS, $\mathrm{pH} 7.4$ ), followed by $4 \%$ paraformaldehyde and $1 \%$ glutaraldehyde ( $\mathrm{n}=3$ per group). The parietal lobe cortex in the ischemic penumbra selected for analysis was cut into small sections and kept overnight in the same fixative. The sections were then immersed in $1 \%$ osmium tetroxide for $2 \mathrm{~h}$, dehydrated in graded ethanol, and embedded in epoxy resin. The samples were then cut into ultrathin sections $(60-70 \mathrm{~nm})$ with an ultramicrotome, poststained with uranyl acetate and lead citrate, and then examined with a transmission electron microscope.

\section{Immunohistochemical staining}

For immunohistochemical studies, formalin-fixed paraffin-embedded tissue sections from adult naked mole rats and adult C57/BL6 mice were dewaxed in xylene and dehydrated. Endogenous peroxidase was blocked by incubating the samples for $10 \mathrm{~min}$ in $3 \% \mathrm{H}_{2} \mathrm{O}_{2}$ at $37{ }^{\circ} \mathrm{C}$, heating for $30 \mathrm{~min}$ at $95{ }^{\circ} \mathrm{C}$ to repair the antigens, and finally rinsing in PBS. The sections were incubated overnight at $4{ }^{\circ} \mathrm{C}$ with the primary antibody, and then with a biotinylated secondary antibody. The primary antibodies used were anti-beclin 1 (1:500; Abcam, Cambridge, UK) and anti-LC3 (1:1000; Cell Signaling Technology, Shanghai, China). The samples were then incubated with peroxidase-conjugated streptavidin for $15 \mathrm{~min}$. Diaminobenzidine tetrahydrochloride (DAB) was used as the substrate to detect antigen-antibody binding. The sections were counterstained with hematoxylin and observed under a light microscope (Olympus IX71).

RNA isolation and RT-PCR to assess gene expression

Beclin 1 and GAPDH mRNA levels in the livers, lungs, and kidneys from one-day-old naked mole rats and one-day-old C57BL/6 mice were quantified by RT-PCR. Total RNA was extracted with TRIzol Reagent (Life Technologies, Inc.), according to the manufacturer's instructions, quantified, and its purity assessed with $A_{260} / A_{280}$ absorption. First-strand cDNA was generated from total RNA $(2 \mu \mathrm{g})$ with the TIANScript cDNA First-Strand Kit (Tiangen, Beijing, China). The transcript expression levels were quantified with the StepOnePlus Real Time PCR Detection Systems (Applied Biosystems, Warrington, UK) using the SYBR® Green Master Mix (Applied Biosystems). Beclin 1 transcript levels were then normalized to the expression of glyceraldehyde-3-phosphate dehydrogenase (GAPDH) transcripts. The rat primer sequences were as follows: for naked mole rat beclin 1, 5'- GTTCAAAGAGGAGGTGGAGAAG-3' and 5'- GAGGAAACCCAGGCAAGAC-3'; for naked mole rat GAPDH, 5'-CGCCTGCTTCACCACCTT-3' and 5'- CCTGCCGCCTGGAGAAA-3'; for mouse beclin 1, 5'- ATGGAGGGGTCTAAGGCGTC-3' and 5'- TGGGCTGTGGTAAGTAATGGA-3'; for mouse GAPDH, 5'-CATGGCCTTCCGTGTTCCTA-3' and 5'- GCGGCACGTCAGATCCA-3'.

To allow the comparison of mRNA expression, the real-time PCR data were analyzed with the $\Delta \Delta \mathrm{Ct}$ method and normalized to the amount of GAPDH cDNA, the endogenous control.

\section{Cell culture and reagents}

Hepatic stellate cells from primary naked mole rats were isolated using methods similar to those reported previously [13]. All primary hepatic stellate cells were maintained at $37^{\circ} \mathrm{C}$ in Dulbecco's modified Eagle's low-glucose medium (Gibco, Invitrogen) supplemented with 10\% fetal bovine serum (Gibco), 100 units $/ \mathrm{mL}$ penicillin, and $100 \mathrm{mg} / \mathrm{mL}$ streptomycin in a humidified incubator under $92 \% \mathrm{~N}_{2}, 5 \% \mathrm{CO}_{2}$, and $3 \%$ $\mathrm{O}_{2}$. DMSO and 3-MA were purchased from Sigma Aldrich. Rapamycin was purchased from Sangon Biotech Co., Ltd (Shanghai, China). All cell lines were used at an early passage ( $<9-12$ population doublings). 


\begin{tabular}{|c|c|c|}
\hline Cellular Phy & Cell Physiol Biochem 2014;33:321-332 & \\
\hline and Biochemistry & $\begin{array}{l}\text { DOI: } 10.1159 / 000356672 \\
\text { Publisned onIIne: rebruary } 05,2014\end{array}$ & $\begin{array}{l}\text { (o } 2014 \text { S. Karger AG, Basel } \\
\text { www.karger.com/cpb }\end{array}$ \\
\hline
\end{tabular}

\section{Cell Counting Kit-8}

The viable cell mass was measured in a Cell Counting Kit-8 (Dojindo, Japan). First, the hepatic stellate cells were seeded at $5 \times 10^{3}$ cell/well in a 96-well flat-bottomed plate, and incubated for up to five days after the addition of rapamycin $(1,20,50$, or $100 \mathrm{nM})$ or 3-MA $(5,10$, or $20 \mathrm{mM})$. The cells were counted at 24,72 , 144 , and $192 \mathrm{~h}$ after treatment. Four replicates were assessed for each treatment. As soon as the treatment was complete, $10 \mathrm{~mL}$ of the Cell Counting Kit-8 solution was added to each well. The plates were incubated for $2 \mathrm{~h}$ in a humidified $\mathrm{CO}_{2}$ incubator at $37^{\circ} \mathrm{C}$. Finally, the absorbance of the samples taken from each well were measured on a microplate reader (Synergy HT, Bio-Tek) at $450 \mathrm{~nm}$. The results were plotted as the percentage of surviving cells in each treatment group relative to those in the untreated group. Sample data are presented as the average of at least three separate experiments.

\section{MDC and DAPI labeling}

The hepatic stellate cells were cultured until day 4, and exposed to $50 \mathrm{nM}$ rapamycin or $20 \mathrm{mM} 3-\mathrm{MA}$ for $24 \mathrm{~h}, 48 \mathrm{~h}$, or $96 \mathrm{~h}$. At the end of the treatments, the cells were stained with monodansylcadaverine (MDC; Sigma, Oakville, CA) and immediately analyzed with fluorescence microscopy. Images were taken with a $380 \mathrm{~nm}$ excitation filter and a $525 \mathrm{~nm}$ emission filter. The hepatic stellate cells were fixed in 96-well plates by incubating the cells with $4 \%$ paraformaldehyde for $10 \mathrm{~min}$ at $37^{\circ} \mathrm{C}$, after which they were stained with 4',6-diamidino-2-phenylindole (DAPI) for $30 \mathrm{~min}$ and observed under a fluorescence microscope (Olympus IX71).

\section{Cell apoptosis assay}

To induce autophagy, the cells were treated with or without rapamycin. 3-MA was used in the experiments to block autophagy. The hepatic stellate cells $\left(2 \times 10^{5}\right.$ cell/well $)$ were cultured in sixwell plates to $70 \%-80 \%$ confluence, and treated with $50 \mathrm{nM}$ rapamycin or $20 \mathrm{mM} 3-\mathrm{MA}$ for $24 \mathrm{~h}$ under hypoxic $\left(3 \% \mathrm{O}_{2}\right)$ conditions. An annexin V-fluorescein isothiocyanate (FITC) assay was used to measure the apoptotic cells by flow cytometry, according to the manufacturer's instructions (Nanjing Keygen Biotech). Briefly, the cells were collected by trypsinization, washed twice with ice-cold PBS, and resuspended in $300 \mu \mathrm{L}$ of $1 \times$ binding buffer, containing $5 \mu \mathrm{L}$ of annexin $\mathrm{V}$ and 5 $\mu \mathrm{L}$ of propidium iodide (PI), for $30 \mathrm{~min}$ at room temperature in the dark. After incubation, at least 10,000 cells were measured on a BD FACSAria flow cytometer (Becton Dickinson). The results were expressed as the percentage of early apoptotic cells (PI-negative and annexin-V-positive cells). Apoptosis was detected based on the nuclear morphology of the cells stained with DAPI, and the cells with condensed and fragmented nuclei were identified as apoptotic.

\section{Western blot analysis}

The hepatic stellate cells were seeded in six-well plates at a density of $5 \times 10^{5}$ cell/well and treated with $50 \mathrm{nM}$ rapamycin or $20 \mathrm{mM} 3-\mathrm{MA}$ for $12 \mathrm{~h}$ or $24 \mathrm{~h}$. At the end of the designated treatments, the naked mole rat hepatic stellate cells were lysed in RIPA lysis buffer with $1 \mathrm{mM}$ phenylmethanesulfonyl fluoride. Equal amounts of protein were separated electrophoretically with SDS-PAGE and transferred to nitrocellulose membrane. The proteins were analyzed as previously described.

\section{Statistical analysis}

SPSS version 12.0 was used for all statistical analyses. All the data and results were confirmed in at least three independent experiments, unless otherwise stated. The statistical significance of the differences between means was assessed with the least significant difference (LSD) $t$ test. $P$ values of $<0.05$ were considered statistically significant.

\section{Results}

One-day-old naked mole rats displayed higher levels of autophagy than one-day-old C57BL/6 mice

We evaluated the autophagy in mice and naked mole rats by measuring the LC3-II and beclin 1 levels in tissue lysates obtained from these animals. The results showed that tissues 


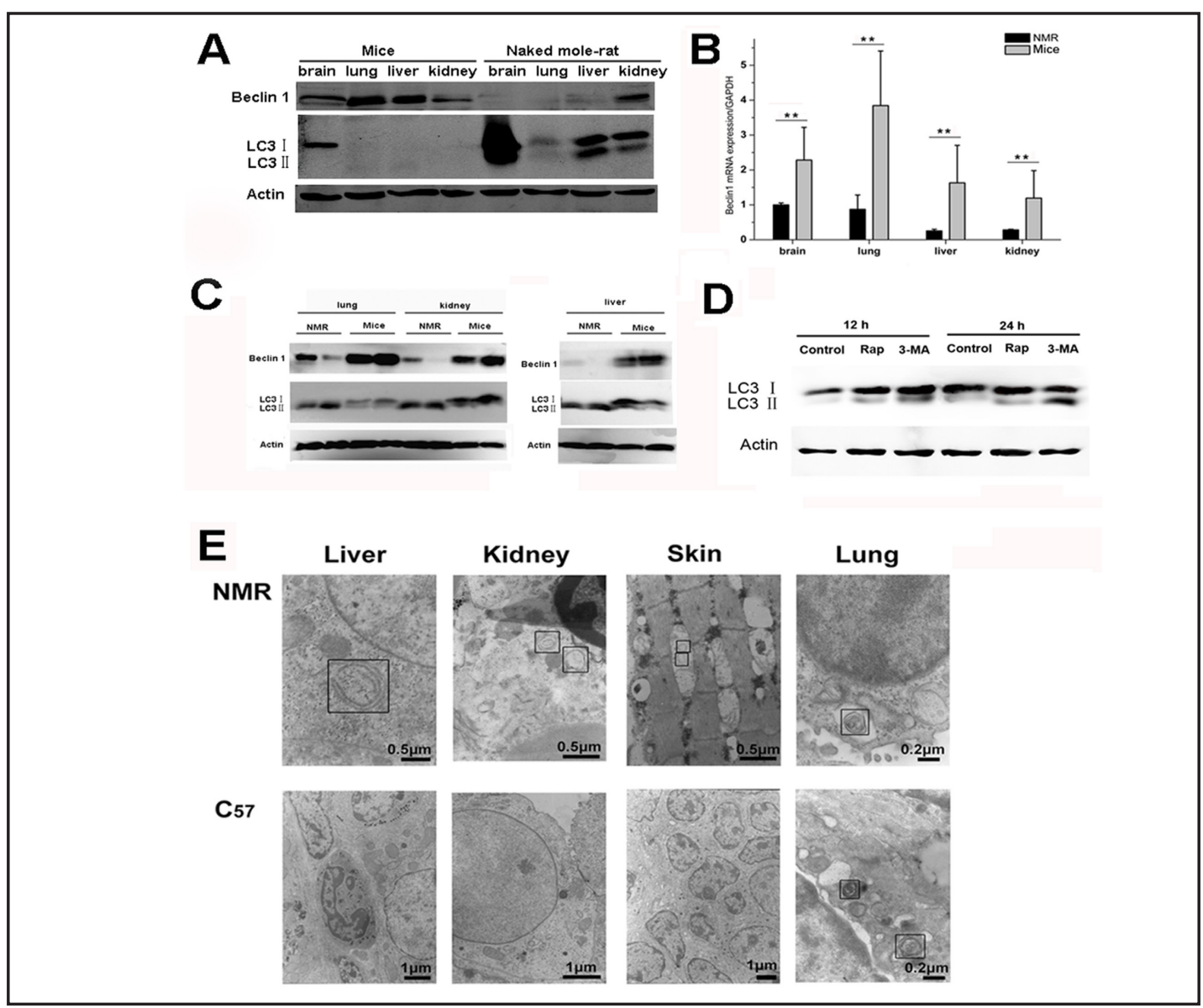

Fig. 1. Western blot analysis and electron microscopic monitoring of autophagy. (A) Western blotting detected LC3 and beclin 1 in one-day-old mouse and one-day-old naked mole rat tissues, including the brain, lung, liver, and kidney. $\beta$-Actin was used as the loading control. (B) Quantitative analysis of beclin 1 expression with real-time RT-PCR. As observed in the western blot analysis, the expression of beclin 1 tended to be lower in one-day-old naked mole rat tissues than in one-day-old mouse tissues. ${ }^{* *} P<0.01$. (C) Expression of beclin 1 and LC3 in adult mouse and adult naked mole rat tissues, including the lung, liver, and kidney. (D) Naked mole rat hepatic stellate cells were treated with $50 \mathrm{nM}$ rapamycin or $20 \mathrm{mM} 3-\mathrm{MA}$ for $12 \mathrm{~h}$ or 24 h. Whole-cell lysates were subjected to western blot analysis for LC3. (E) Representative electron micrographs showing autophagic vacuoles in different organs from one-day-old naked mole rats and one-day-old C57BL/ 6 mice. Black arrows indicate the autophagic vacuoles.

from one-day-old naked mole rats had accumulated more LC3-II than the tissues from oneday-old C57BL/6 mice (Fig. 1A). Electron microscopic analysis also demonstrated that a higher number of double-membraned autophagosomes were present in one-day-old naked mole rat organs than in one-day-old C57BL/6 mouse organs (liver, lung, kidney, and skin; Fig. 1E). Tissue specimens from adult naked mole rats also had significantly higher levels of LC3-II than adult C57BL/6 mouse tissues (Fig. 1C). Although the quantity of LC3-I was too low to be seen in Fig. 1C, it was detected exclusively by increasing the sample volume (data not shown). Tissue specimens were stained for LC3 immunoreactivity, as described in the Methods. LC3 immunoreactivity was significantly stronger in the adult naked mole rats than in the adult C57BL/6 mice (Fig. 2B). Autophagy is characterized by the formation of autophagosomes and autolysosomes. Taken together, these data suggest that the naked mole rats maintain higher levels of autophagy than mice under basal conditions, before it can negatively affects organ functionality. 


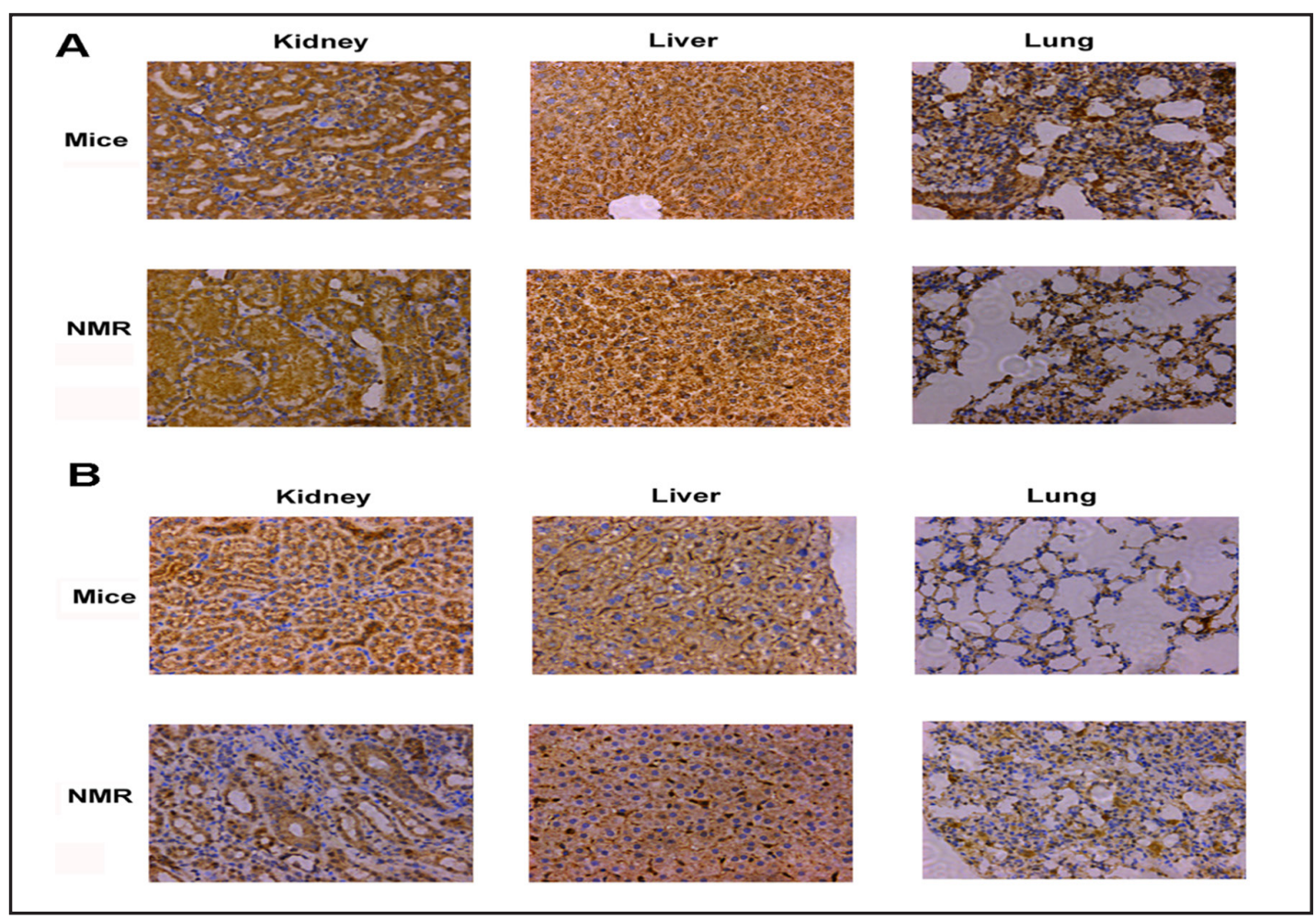

Fig. 2. Immunohistochemical photomicrographs of LC3 and beclin 1 in different organs from adult naked mole rats and adult C57BL/6 mice (ESCC) (×200). (A) Beclin 1 immunoreactivity in different organs from C57BL/6 mice and naked mole rats; (B) LC3 and (D) beclin 1 immunoreactivity in different organs from C57BL/6 mice and naked mole rats. LC3 positivity was apparent as brown staining in the cytoplasm and occasionally in the nuclei.

Fig. 3. Dose-response curves and time courses of rapamycin- and 3-MAinduced growth inhibition in naked mole rat hepatic stellate cells. (A) Effects of rapamycin on the growth of naked mole rat hepatic stellate cells. (B) Effects of 3-MA on the growth of naked mole rat hepatic stellate cells.
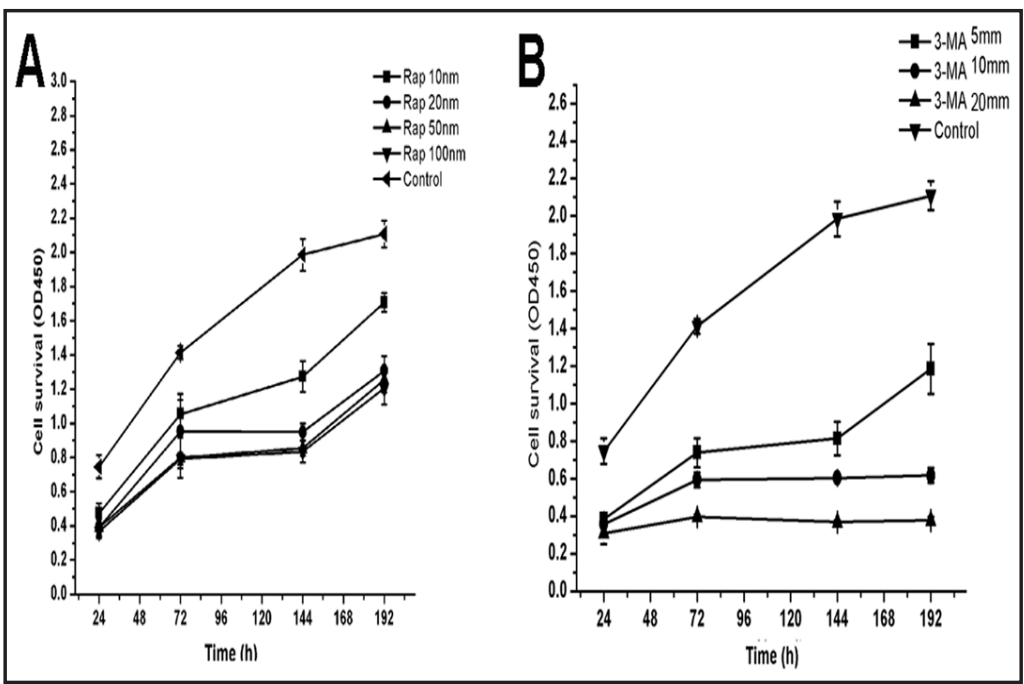

Beclin 1 expression was lower in naked mole rat tissues than in tissues from C57BL/6 mice

The beclin 1 expression in one-day-old naked mole rat tissues (liver, lung, kidney, and brain) was even lower than in one-day-old mouse tissues (Fig. 1A). To confirm these findings, beclin 1 mRNA levels were determined with real-time PCR. The mean transcript levels of beclin 1 were markedly lower in one-day-old naked mole rat tissues than in one-day-old C57BL/6 mouse tissues (Fig. 1B). Similarly, beclin 1 protein levels were lower in adult naked 
Fig. 4. Visualization of autophagy activation with monodansylcadaverine (MDC) in naked mole rat hepatic stellate cells. Naked mole rat hepatic stellate cells were treated with $50 \mathrm{nM}$ rapamycin or 20 mM 3-MA and incubated for 24,48 , or $96 \mathrm{~h}$. The cells were stained with MDC, as described in the Materials and Methods (all panels, $\times 200$ magnification).

Fig. 5. Detection of apoptosis with DAPI staining. Fluorescence microscopy was used to observe the cell morphology following DAPI staining (all panels, $\times 200$ magnification).
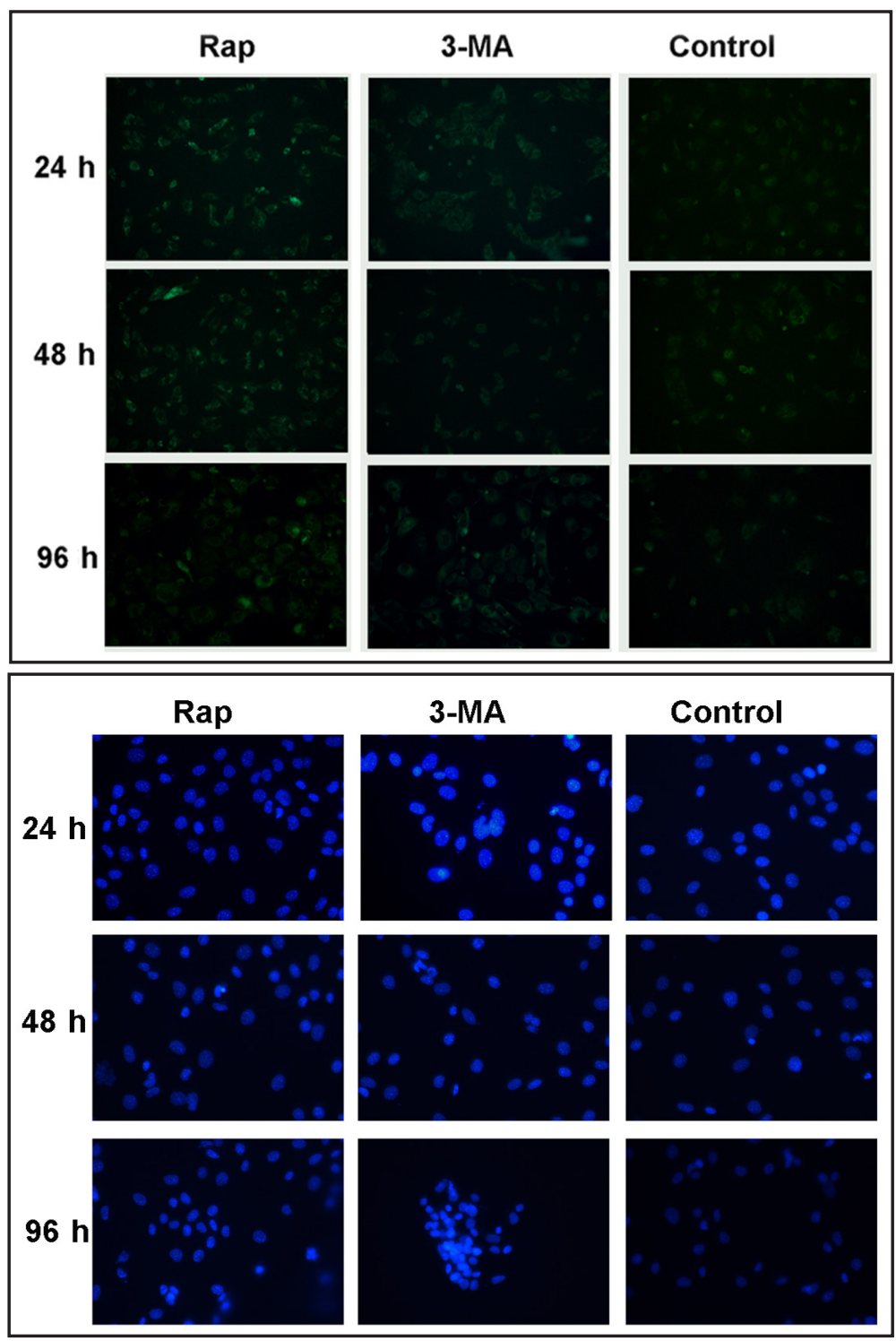

mole rat tissues than in adult C57BL/6 mouse tissues (Fig. 1C). The beclin 1 expression in the naked mole rat and C57BL/6 mouse tissues showed high cytoplasmic and low nuclear patterns (Fig. 2A).

\section{Autophagy in different organs}

We measured the levels of autophagy in different organs (liver, lung, kidney, and brain). LC3-II levels were higher in the brains of one-day-old naked mole rats than in their other organs (Fig. 1A). Thus, the level of autophagy was higher in the brain than in the liver, lung, or kidney.

Rapamycin treatment induced apoptosis in naked mole rat hepatic stellate cells and increased the expression of autophagy markers

Figure 1D illustrates the effects of rapamycin treatment on the expression levels of LC3-II in hepatic stellate cells. Treatment with rapamycin for $12 \mathrm{~h}$ or $24 \mathrm{~h}$ led to the accumulation of LC3-II (Fig. 1D). MDC is an autofluorescent compound that has been proposed for use as a tracer of autophagic vacuoles. We studied the incorporation of MDC into hepatic stellate cells using fluorescence microscopy. As shown in Figure 4, hepatic stellate 


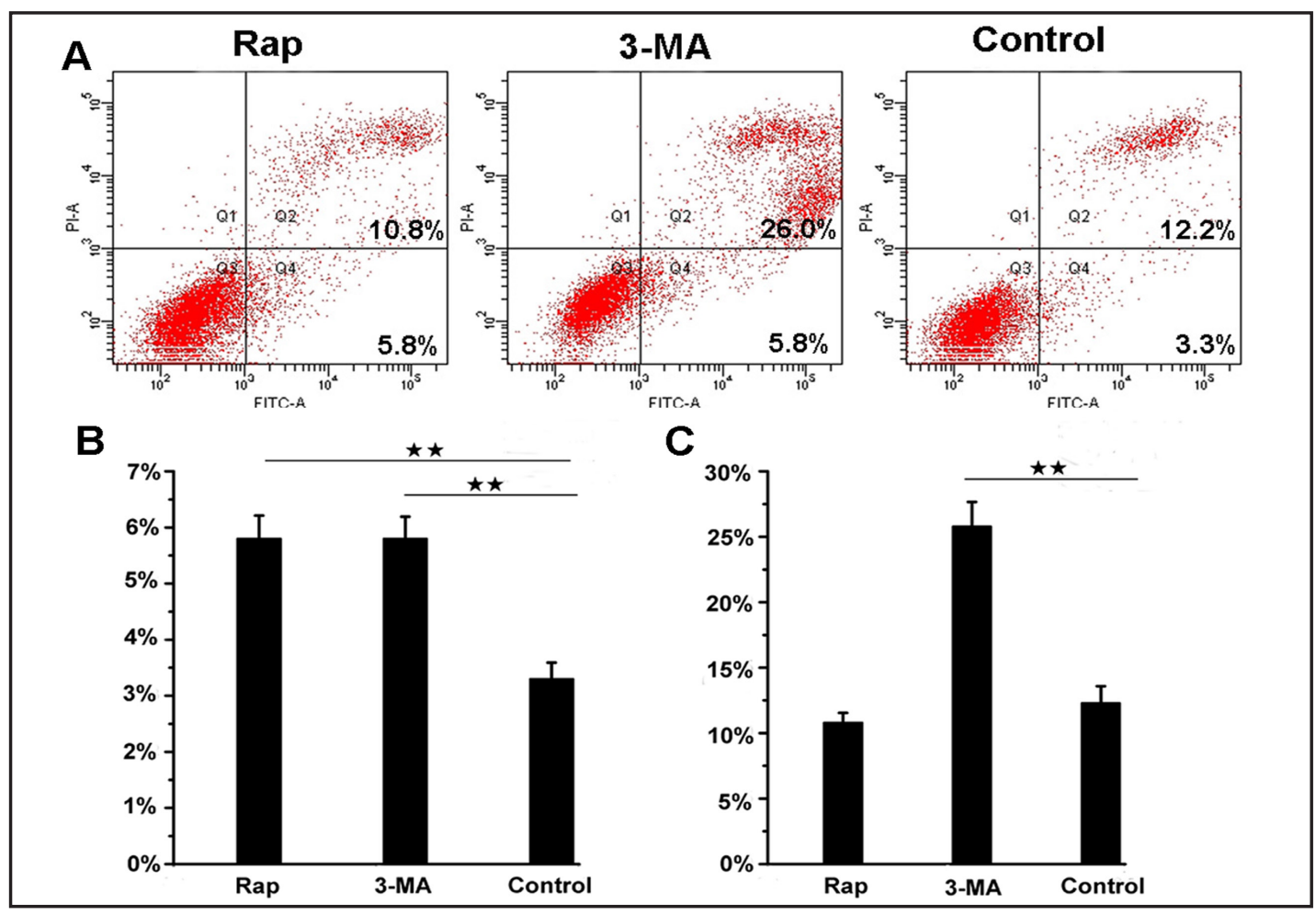

Fig. 6. Rapamycin and 3-MA induce apoptosis in naked mole rat hepatic stellate cells. (A) Naked mole rat hepatic stellate cells were treated with $50 \mathrm{nM}$ rapamycin or $20 \mathrm{mM}$ 3-MA for $48 \mathrm{~h}$. The negative control was treated with PBS. Apoptosis was analyzed with annexin V and PI staining and flow cytometry. (B) Graph indicates the percentage of annexin-V-positive and PI-negative cells, which are early apoptotic cells (means \pm SD). (C) Graph indicates the percentage of annexin-V-positive and PI- positive cells, which are late apoptotic cells. ${ }^{* *} P<0.01$.

cells that were treated with rapamycin for 24,48 , or $96 \mathrm{~h}$ demonstrated a punctate pattern of MDC-labeled fluorescence. The control cells showed low basal levels of apoptosis (3.110 \pm $0.384 \%$ ). However, a significantly higher rate of apoptosis was observed following treatment with $50 \mathrm{nM}$ rapamycin for $48 \mathrm{~h}(P<0.05$; Fig. 6A, 6B). These data indicate that rapamycin disrupts the cellular mechanisms that regulate autophagy and apoptosis. Rapamycin, which is a mammalian TORC1 (mTORC1) inhibitor and autophagy activator, was administered daily at various concentrations in the range of $10-100 \mathrm{nM}$, at different time intervals $(24,72$, 144 , and $192 \mathrm{~h}$ ). Cell counting revealed that rapamycin caused dose-dependent cell growth inhibition (Fig. 3A).

Prolonged treatment with 3-MA induced the accumulation of the autophagy marker LC3II

Phosphatidylinositide 3-kinase inhibitors, such as 3-MA, have been widely used to inhibit autophagy. To our surprise, we found that prolonged treatment with 3-MA induced significant LC3-II enrichment in naked mole rat hepatic stellate cells (Fig. 1D). The number of cells was significantly lower in the 3-MA-treated cells than in the control cells (Fig. 3B). Hepatic stellate cells treated with 3-MA for 24,48 , or $96 \mathrm{~h}$ demonstrated a punctate pattern of MDC-labeled fluorescence (Fig. 4). DAPI staining showed clear evidence of condensed and shrunken nuclei in the 3-MA-treated cells (Fig. 5). In the annexin-V-FITC assay, 14\% more cells in the 3-MA-treated group were annexin-V-positive and PI-positive, indicative of 
late apoptotic cells (Fig. 6A, 6C). A similar trend was observed in the number of annexin-Vpositive and PI-negative cells, indicative of early apoptotic cells (Fig. 6A, 6B). The apoptotic ratio in the 3-MA-treated cells was significantly higher than that in the control group. Cell counts revealed that 3-MA also produced dose-dependent cell growth inhibition (Fig. 3B).

\section{Discussion}

Autophagy is the cellular process that mediates the lysosomal degradation of longlived cytoplasmic proteins, initiated under conditions of cell differentiation, starvation, or stress, including oxidative stress, endoplasmic reticulum stress, and the accumulation of protein aggregates [14-17]. Autophagy is probably also a survival response to unfavorable conditions, such as the depletion of growth factors, hypoxia, endoplasmic reticulum stress, microbial infection, and diseases characterized by the accumulation of protein aggregates [18]. The amino acids produced during autophagy can be used to synthesize proteins that are important for the organism's adaptation to nutrient-deficient environments [14]. Wang et al. [19] found that fibroblasts from long-lived mutant mice displayed increased autophagy and lower TOR activity after nutrient deprivation or oxidative stress. Studies of senescent avian and mammalian fibroblasts have shown that cells from long-lived birds maintain autophagosomal and lysosomal enzymes better than mammalian cell lines, in which these activities are downregulated with age $[20,21]$. These findings indicate that autophagy helps to maintain cellular homeostasis and cell survival [22], and plays a significant role in the determination of lifespan in the naked mole rat. The naked mole rat is not an inbred strain, but breeding occurs between related individuals. C57BL/ 6 mice are a standard inbred strain, and have low susceptibility to tumors [23, 24]. Therefore, the inbred C57BL/6 mouse strain was used in this comparative study.

Once autophagy is initiated, cytoplasmic material becomes enclosed in a doublemembrane structure, which subsequently fuses with a lysosome, leading to the degradation of damaged or unwanted cell components. Here, we showed that higher levels of LC3-II accumulated in the organs of one-day-old naked mole rats than in the organs of short-lived mice. However, LC3 expression did not differ significantly between the adult naked mole rats (eight months old) and the adult C57BL/6 mice (eight weeks old). Therefore, both the adult naked mole rat and adult C57BL/6 mouse have high basal levels of autophagy. These results indicate that the naked mole rat maintains high levels of autophagy under basal conditions in adulthood and childhood. Rodriguez et al. [25] showed that macroautophagy is substantially higher in naked mole rat fibroblasts maintained under serum starvation than in fibroblasts from shorter-lived mice. Similarly, when autophagy was assessed by monitoring a marker of vacuole development (i.e., the conversion of LC3-I to LC3-II), the LC3-II/LC3-I ratio induced by serum deprivation was approximately two-fold higher in the naked mole rat cells [26]. These data suggest that nutrient deprivation plays an important role in the maintenance of protein quality in naked mole rats. However, we found that the expression of beclin 1 in their normal tissues (liver, lung, kidney, and brain) was even lower than that in the tissues of short-lived mice (Fig. 1A and 1B). Beclin 1 expression in the livers, lungs, and kidneys of C57BL/6 mice was normal, similar to that reported in other studies. This indicates that the LC3-II level usually correlates well with the number of autophagosomes, and can be used to monitor autophagosome formation. Although beclin 1 plays a critical role in the regulation of autophagosome formation, it is also involved in the formation of preautophagosomal structures. The amount of beclin 1 did not correlate well with the number of autophagosomes.

3-Methyladenine (3-MA) has been known to selectively and potently inhibit autophagydependent protein degradation and to suppress the formation of autophagosomes [27, 28]. To our surprise, 3-MA promoted autophagy when naked mole rat hepatic stellate cells were treated with it for a prolonged period. A similar result has already been reported by Wu et al. [29]. They showed that prolonged treatment with 3-MA (up to $9 \mathrm{~h}$ ) in complete medium induced autophagy in mouse embryonic fibroblasts, L929 mouse fibrosarcoma cells, and 
human renal epithelial 293T cells [29]. These results demonstrate that 3-MA plays a dual role in the modulation of autophagy, promoting autophagy or suppressing it.

Rapamycin is a powerful mTOR inhibitor and strong inducer of autophagy. The activation of autophagy may lead either to cell differentiation or to cell death [21, 30-33]. Autophagy is clearly implicated in cell differentiation and cell death because low doses of rapamycin activate autophagy [34]. At a concentration of $50 \mathrm{nM}$, rapamycin inhibited the growth of naked mole rat hepatic stellate cells. 3-MA-induced autophagy can facilitate selenite-induced apoptosis in hepatic stellate cells. We investigated the effects of these compounds on the apoptosis of hepatic stellate cells. Our flow cytometry results are consistent with this finding. When hepatic stellate cells were treated with 3-MA, the apoptotic ratio was significantly increased relative to that of the control group. Considering these data together, naked mole rat hepatic stellate cells have the appropriate levels of autophagic flux to promote cellular homeostasis, stress responsiveness, and cell survival. Excessive autophagy, such as that induced by rapamycin or 3-MA, is maladaptive and may possibly trigger cell death.

The genetic screening of yeast (in macroautophagy research) and novel biochemical approaches (in chaperone-mediated autophagy research) have helped to identify a subset of genes and protein products that act as effectors or modulators of these autophagic pathways. Manipulation in these genes has confirmed the tight association between autophagy, lifespan, and aging [35]. Functional autophagy has also been shown to be necessary to attain the maximal lifespan extension, mediated by the deletion of other genes, such as those encoding mTOR [5] or tumor suppressor p53 [36], which curiously represses macroautophagy in mammals [37]. Liu confirmed that beclin 1 controls p53 levels by regulating the deubiquitination activity of USP10 and USP13 [38]. Our unpublished data revealed that p53 expression in normal naked mole rat tissues (liver, lung, kidney, and brain) is even lower than that in the tissues of short-lived mice (Lin, unpublished data). Furthermore, beclin 1 expression is very low in normal naked mole rat tissues. The mechanism of beclin 1 activity as a tumor suppressor remains to be clarified, and further studies are required to explore this mechanism in detail.

In conclusion, our results indicate that the rate of autophagy is higher in young naked mole rats than in young shorter-lived mice, and different levels of autophagy occur in different organs. Our study also shows that treatment with 3-MA or rapamycin increases the ratio of apoptotic cells to normal hepatic stellate cells. In the next study, we will investigate whether autophagy facilitates the survival of naked mole rat hepatic stellate cells.

\section{Acknowledgments}

This work was supported by a grant from the Shanghai Committee of Science and Technology, China (no. 10140900800).

\section{References}

$\checkmark 1$ Larson J, Park TJ: Extreme hypoxia tolerance of naked mole-rat brain. Neuroreport 2009;20:16341637.

2 Tang H, Da L, Mao Y, Li Y, Li D, Xu Z, Li F, Wang Y, Tiollais P, Li T, Zhao M: Hepatitis B virus X protein sensitizes cells to starvation-induced autophagy via up-regulation of beclin 1 expression. Hepatology 2009;49:60-71.

-3 Melendez A, Talloczy Z, Seaman M, Eskelinen EL, Hall DH, Levine B: Autophagy genes are essential for dauer development and life-span extension in C. elegans. Science 2003;301:1387-1391.

4 Hars ES, Qi H, Ryazanov AG, Jin S, Cai L, Hu C, Liu LF:Autophagy regulates ageing in C. elegans. Autophagy 2007;3:93-95.

-5 Hansen M, Chandra A, Mitic LL, Onken B, Driscoll M, Kenyon C: A role for autophagy in the extension of lifespan by dietary restriction in C. elegans. PLoS Genet 2008;4:e24. 
6 Buffenstein R, Jarvis JU: The naked mole rat--a new record for the oldest living rodent. Sci Aging Knowledge Environ 2002;2002:pe7.

7 Wang Y, Han R, Liang ZQ, Wu JC, Zhang XD, Gu ZL, Qin ZH: An autophagic mechanism is involved in apoptotic death of rat striatal neurons induced by the non-N-methyl-D-aspartate receptor agonist kainic acid. Autophagy 2008;4:214-226.

-8 Kabeya Y, Mizushima N, Ueno T, Yamamoto A, Kirisako T, Noda T, Kominami E, Ohsumi Y, Yoshimori T: LC3, a mammalian homologue of yeast Apg8p, is localized in autophagosome membranes after processing. EMBO J 2000;19:5720-5728.

-9 Klionsky DJ, Abeliovich H, Agostinis P, Agrawal DK, Aliev G, Askew DS, Baba M, Baehrecke EH, Bahr BA, Ballabio A, Bamber BA, Bassham DC, Bergamini E, Bi X, Biard-Piechaczyk M, Blum JS, Bredesen DE, Brodsky JL, Brumell JH, Brunk UT, Bursch W, Camougrand N, Cebollero E, Cecconi F, Chen Y, Chin LS, Choi A, Chu CT, Chung J, Clarke PG, Clark RS, Clarke SG, Clavé C, Cleveland JL, Codogno P, Colombo MI, Coto-Montes A, Cregg JM, Cuervo AM, Debnath J, Demarchi F, Dennis PB, Dennis PA, Deretic V, Devenish RJ, Di Sano F, Dice JF, Difiglia M, Dinesh-Kumar S, Distelhorst CW, Djavaheri-Mergny M, Dorsey FC, Dröge W, Dron M, Dunn WA Jr, Duszenko M, Eissa NT, Elazar Z, Esclatine A, Eskelinen EL, Fésüs L, Finley KD, Fuentes JM, Fueyo J, Fujisaki K, Galliot B, Gao FB, Gewirtz DA, Gibson SB, Gohla A, Goldberg AL, Gonzalez R, González-Estévez C, Gorski S, Gottlieb RA, Häussinger D, He YW, Heidenreich K, Hill JA, Høyer-Hansen M, Hu X, Huang WP, Iwasaki A, Jäättelä M, Jackson WT, Jiang X, Jin S, Johansen T, Jung JU, Kadowaki M, Kang C, Kelekar A, Kessel DH, Kiel JA, Kim HP, Kimchi A, Kinsella TJ, Kiselyov K, Kitamoto K, Knecht E, Komatsu M, Kominami E, Kondo S, Kovács AL, Kroemer G, Kuan CY, Kumar R, Kundu M, Landry J, Laporte M, Le W, Lei HY, Lenardo MJ, Levine B, Lieberman A, Lim KL, Lin FC, Liou W, Liu LF, Lopez-Berestein G, López-Otín C, Lu B, Macleod KF, Malorni W, Martinet W, Matsuoka K, Mautner J, Meijer AJ, Meléndez A, Michels P, Miotto G, Mistiaen WP, Mizushima N, Mograbi B, Monastyrska I, Moore MN, Moreira PI, Moriyasu Y, Motyl T, Münz C, Murphy LO, Naqvi NI, Neufeld TP, Nishino I, Nixon RA, Noda T, Nürnberg B, Ogawa M, Oleinick NL, Olsen LJ, Ozpolat B, Paglin S, Palmer GE, Papassideri I, Parkes M, Perlmutter DH, Perry G, Piacentini M, Pinkas-Kramarski R, Prescott M, Proikas-Cezanne T, Raben N, Rami A, Reggiori F, Rohrer B, Rubinsztein DC, Ryan KM, Sadoshima J, Sakagami H, Sakai Y, Sandri M, Sasakawa C, Sass M, Schneider C, Seglen PO, Seleverstov O, Settleman J, Shacka JJ, Shapiro IM, Sibirny A, Silva-Zacarin EC, Simon HU, Simone C, Simonsen A, Smith MA, Spanel-Borowski K, Srinivas V, Steeves M, Stenmark H, Stromhaug PE, Subauste CS, Sugimoto S, Sulzer D, Suzuki T, Swanson MS, Tabas I, Takeshita F, Talbot NJ, Tallóczy Z, Tanaka K, Tanaka K, Tanida I, Taylor GS, Taylor JP, Terman A, Tettamanti G, Thompson CB, Thumm M, Tolkovsky AM, Tooze SA, Truant R, Tumanovska LV, Uchiyama Y, Ueno T, Uzcátegui NL, van der Klei I, Vaquero EC, Vellai T, Vogel MW, Wang HG, Webster P, Wiley JW, Xi Z, Xiao G, Yahalom J, Yang JM, Yap G, Yin XM, Yoshimori T, Yu L, Yue: ::Z,:Yuzaki M, Zabirnyk 0, Zheng X, Zhu $\mathrm{X}$, Deter RL: Guidelines for the use and interpretation of assays for monitoring autophagy in higher eukaryotes. Autophagy 2008;4:151-175.

-10 Kabeya Y, Mizushima N, Yamamoto A, Oshitani-Okamoto S, Ohsumi Y, Yoshimori T: LC3, GABARAP and GATE16 localize to autophagosomal membrane depending on form-II formation. J Cell Sci 2004;117:2805-2812.

-11 Friedman SL: Hepatic stellate cells: protean, multifunctional, and enigmatic cells of the liver. Physiol Rev 2008;88:125-172.

12 Klionsky DJ, Abdalla FC, Abeliovich H, et al. Guidelines for the use and interpretation of assays for monitoring autophagy. Autophagy 2012;8:445-544.

13 Ramm GA: Isolation and culture of rat hepatic stellate cells. J Gastroenterol Hepatol 1998;13:846-851.

14 Mizushima N: Autophagy: process and function. Genes Dev 2007;21:2861-2873.

-15 Yorimitsu T, Nair U, Yang Z, Klionsky DJ: Endoplasmic reticulum stress triggers autophagy. J Biol Chem 2006;281:30299-30304.

-16 Sarkar S, Perlstein EO, Imarisio S, Pineau S, Cordenier A, Maglathlin RL, Webster JA, Lewis TA, O'Kane CJ, Schreiber SL, Rubinsztein DC: Small molecules enhance autophagy and reduce toxicity in Huntington's disease models. Nat Chem Biol 2007;3:331-338.

17 Gao L, Jiang T, Guo J, Liu Y, Cui G, Gu L, Su L, Zhang Y: Inhibition of autophagy contributes to ischemic postconditioning-induced neuroprotection against focal cerebral ischemia in rats. PLoS One 2012;7:e46092. 
Zhao et al.: Autophagy Affects Apoptosis of Hepatic Stellate Cells

18 Kourtis N, Tavernarakis N: Autophagy and cell death in model organisms. Cell Death Differ 2009;16:21-30.

-19 Wang M, Miller RA: Fibroblasts from long-lived mutant mice exhibit increased autophagy and lower TOR activity after nutrient deprivation or oxidative stress. Aging Cell 2012;11:668-674.

20 Massey AC, Kiffin R, Cuervo AM: Autophagic defects in aging: looking for an "emergency exit"? Cell Cycle 2006;5:1292-1296.

-21 Zhao Y, Huang Q, Yang J, Lou M, Wang A, Dong J, Qin Z, Zhang T: Autophagy impairment inhibits differentiation of glioma stem/progenitor cells. Brain Res 2010;1313:250-258.

-22 Reggiori F, Klionsky DJ: Autophagy in the eukaryotic cell. Eukaryot Cell 2002;1:11-21.

- 23 Stanley LA, Devereux TR, Foley J, Lord PG, Maronpot RR, Orton TC, Anderson MW:Proto-oncogene activation in liver tumors of hepatocarcinogenesis-resistant strains of mice. Carcinogenesis 1992;13:2427-2433.

-24 Kushida M, Kamendulis LM, Peat TJ, Klaunig JE: Dose-related induction of hepatic preneoplastic lesions by diethylnitrosamine in C57BL/6 mice. Toxicol Pathol 2011;39:776-786.

-25 Rodriguez KA, Wywial E, Perez VI, Lambert AJ, Edrey YH, Lewis KN, Grimes K, Lindsey ML, Brand MD, Buffenstein R:. Walking the oxidative stress tightrope: a perspective from the naked mole-rat, the longest-living rodent. Curr Pharm Des 2011;17:2290-2307.

-26 Strecker V, Mai S, Muster B, Beneke S, Bürkle A, Bereiter-Hahn J, Jendrach M: Aging of different avian cultured cells: lack of ROS-induced damage and quality control mechanisms. Mech Ageing Dev 2010;131:48-59.

27 Bursch W: The autophagosomal-lysosomal compartment in programmed cell death. Cell Death Differ 2001;8:569-581.

-28 Seglen PO, Gordon PB: 3-Methyladenine: specific inhibitor of autophagic/lysosomal protein degradation in isolated rat hepatocytes. Proc Natl Acad Sci U S A 1982;79:1889-1892.

-29 Wu YT, Tan HL, Shui G, Bauvy C, Huang Q, Wenk MR, Ong CN, Codogno P, Shen HM: Dual role of 3-methyladenine in modulation of autophagy via different temporal patterns of inhibition on class I and III phosphoinositide 3-kinase. J Biol Chem 2010;285:10850-10861.

-30 Park MA, Reinehr R, Haussinger D, Voelkel-Johnson C, Ogretmen B, Yacoub A, Grant S, Dent P: Sorafenib activates CD95 and promotes autophagy and cell death via Src family kinases in gastrointestinal tumor cells. Mol Cancer Ther 2010;9:2220-2231.

- 31 Liu WT, Lin CH, Hsiao M, Gean PW: Minocycline inhibits the growth of glioma by inducing autophagy. Autophagy 2011;7:166-175.

-32 Lefranc F, Facchini V, Kiss R: Proautophagic drugs: a novel means to combat apoptosis-resistant cancers, with a special emphasis on glioblastomas. Oncologist 2007;12:1395-1403.

- 33 Arcella A, Biagioni F, Antonietta Oliva M, Bucci D, Frati A, Esposito V, Cantore G, Giangaspero F, Fornai F: Rapamycin inhibits the growth of glioblastoma. Brain Res 2013;1495:37-51.

-34 Pan T, Kondo S, Zhu W, Xie W, Jankovic J, Le W: Neuroprotection of rapamycin in lactacystin-induced neurodegeneration via autophagy enhancement. Neurobiol Dis 2008;32:16-25.

-35 Cuervo AM: Autophagy and aging: keeping that old broom working. Trends Genet 2008;24:604-612.

-36 Tavernarakis N, Pasparaki A, Tasdemir E, Maiuri MC, Kroemer G: The effects of p53 on whole organism longevity are mediated by autophagy. Autophagy 2008;4:870-873.

- 37 Crighton D, Wilkinson S, O'Prey J, Syed N, Smith P, Harrison PR, Gasco M, Garrone 0, Crook T, Ryan KM:DRAM, a p53-induced modulator of autophagy, is critical for apoptosis. Cell 2006;126:121-134.

-38 Liu J, Xia H, Kim M, Xu L, Li Y, Zhang L, Cai Y, Norberg HV, Zhang T, Furuya T, Jin M, Zhu Z, Wang H, Yu J, Li Y, Hao Y, Choi A, Ke H, Ma D, Yuan J: Beclin1 controls the levels of p53 by regulating the deubiquitination activity of USP10 and USP13. Cell 2011;147:223-234. 